\title{
TEMA 2017: Cistitis hemorrágica inducida por radioterapia
}
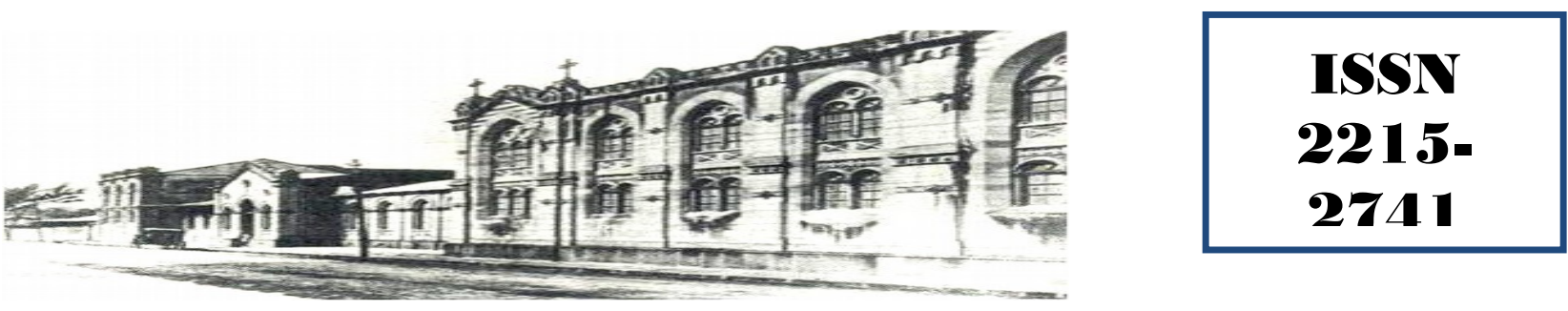

Haspital San quan de Dios. San José. Costa Rica. Fundado en 1845

Recibido:

$20 / 05 / 2017$

Aceptado:

$15 / 06 / 2017$

Santiago Batalla Garrido ${ }^{1}$

Danilo Eduardo Garzona Navas ${ }^{2}$

${ }^{1}$ Médico General. Hospital Nacional de Niños. Correo electrónico: santiago.batalla@gmail.com

${ }^{2}$ Médico especialista en Urología. Hospital Nacional de Geriatría y Gerontología. Correo electrónico: danilogarzona@hotmail.com

\section{RESUMEN}

La cistitis hemorrágica es una complicación que puede surgir luego de utilizar radioterapia para algunos tipos de cáncer pélvicos. Al no ser una entidad frecuente, no existe abundante evidencia de peso con respecto al manejo terapéutico que se debe implementar ante su aparición. Este artículo de revisión pretende dar a conocer con más detalle la fisiopatología detrás de la enfermedad y describir los diferentes tratamientos que se han estudiado.

\section{PALABRAS CLAVE}

Cistitis hemorrágica. Radioterapia. Cáncer pélvico. Hematuria intratable.

\section{ABSTRACT}

Hemorrhagic cystitis is a complication that can arise after radiotherapy for different types of pelvic cancer. Since it does not appear frequently, there isn't an abundance of evidence regarding its management. The aim of this review paper is to present the pathophysiology behind the disease and to describe the therapeutic alternatives that have been studied for it.

\section{KEY WORDS}

Hemorrhagic cystitis. Radiotherapy. Pelvic cancer. Intractable hematuria

\section{INTRODUCCIÓN}

La radioterapia es una modalidad comúnmente utilizada en el manejo de diversos procesos oncológicos pélvicos para atacar células malignas de manera no invasiva. A pesar de emitir dosis individualizadas en zonas muy bien delimitadas, siempre hay riesgo de afectar parte del tejido sano circundante y causar efectos adversos considerables ${ }^{(1-3)}$. Dentro de estas complicaciones se encuentra la cistitis hemorrágica, definida como un sangrado difuso de la mucosa de la vejiga que viene precedido por cambios histopatológicos inducidos por la radiación. La severidad del sangrado puede variar desde hematuria leve hasta hemorragias graves que comprometen la vida del paciente ${ }^{(4,5)}$. Actualmente existen varios fármacos $\mathrm{y}$ procedimientos que se utilizan para el 
tratamiento de esta condición, sin embargo aún no se cuenta con protocolos nacionales o internacionales que guíen la conducta terapéutica ${ }^{(6)}$.

\section{DISCUSIÓN}

\section{Incidencia}

La aparición de la cistitis hemorrágica se ha documentado en un pequeño pero importante porcentaje de la población que recibe radioterapia pélvica, con cifras que rodean entre el $5-10 \%$ de los pacientes ${ }^{(7)}$. Existe también una gran variabilidad en el tiempo transcurrido entre el tratamiento y la manifestación de esta patología. Algunas series han observado casos tan temprano como a los 2 meses y tan tarde como a los 26 años $^{(8,9)}$, con reportes que establecen el promedio entre los 3 y 4 años ${ }^{(7,9)}$. Los hombres tienen mayor probabilidad que las mujeres de desarrollar esta complicación (2.8:1) debido al uso frecuente de la radioterapia como parte del tratamiento del cáncer de próstata ${ }^{(1)}$. Debido al envejecimiento de la población general $\mathrm{y}$ al incremento en el uso de la radioterapia para los diferentes tumores pélvicos, la prevalencia de la cistitis hemorrágica podría ir en aumento a pesar de las mejoras técnicas en la administración de la radiación ${ }^{(6)}$.

\section{Factores de riesgo}

El desarrollo de la enfermedad es multifactorial e incluye tanto elementos preexistentes del paciente como las características tumorales y las técnicas de radioterapia utilizadas ${ }^{(7)}$. Las comorbilidades como la diabetes, los desordenes del tejido conectivo, el fumado, la hipertensión arterial, el uso de anticoagulantes y la historia de cirugía abdominal previa aumentan la potencial toxicidad del tratamiento ${ }^{(6,7,9,10)}$. Así mismo, la probabilidad de que el evento se presente es directamente proporcional a la dosis de radioterapia total, a la dosis por fracción y al volumen de la vejiga que es irradiado ${ }^{(7,9)}$. Adicionalmente, la cirugía, las complicaciones postquirúrgicas y la quimioterapia juegan un papel crítico en la determinación del riesgo de desarrollar esta $\operatorname{morbilidad}^{(7,9,10)}$.

\section{Fisiopatología}

El epitelio vesical normal tiene una tasa de recambio y proliferación baja, lo cual lo hace muy sensible a los efectos de la radiación ${ }^{(11,12)}$. En estos casos, la emisión de energía puede causar muerte celular inmediata o muerte retardada si se llega a afectar directamente la información genética ${ }^{(11,13)}$.

Los síntomas tempranos se han atribuido a la lesión de la capa de glicosaminoglicanos que recubre el epitelio urinario y cuya función principal es la de actuar como barrera. Una vez que se produce el daño, la capa se vuelve permeable y esto permite que la orina irrite e inflame el tejido circundante debido a su carácter hipertónico ${ }^{(8,}{ }^{11)}$. Estos procesos poco a poco causan edema y exfoliación de la mucosa, lo cual expone las capas subyacentes incluyendo al músculo liso y a los vasos que la nutren ${ }^{(6,7)}$.

El edema eventualmente alcanza a la vasculatura endotelial, aproximadamente 3 meses posterior a la lesión inicial, y se da una obliteración progresiva de estos componentes. Consecuentemente se aumenta el daño tisular por la hipoxia provocada y se da la neoformación de vasos frágiles y propensos al sangrado ${ }^{(4,7,8,12,14)}$. Al mismo tiempo, el músculo liso también se edematiza y al ser destruido es reemplazado por fibroblastos que producen una capa de colágeno con la resultante disminución de la distensibilidad y capacidad vesical ${ }^{(1,10,12,15)}$.

El tejido afectado tiene la característica de ser hipovascular, hipocelular e hipóxico, y por lo tanto sufre una disminución en su capacidad de reparación $^{(6,9,12)}$. Estas condiciones aumentan la probabilidad de sufrir ulceraciones y hemorragias, que incluso pueden progresar a necrosis, perforación vesical y formación de fístulas $^{(6,7,9,12,15)}$.

\section{Cuadro clínico}

Los síntomas agudos asociados a la cistitis por radiación comúnmente aparecen de 3-4 semanas posterior al inicio de la terapia y suelen desaparecer entre las 4-6 semanas luego de su conclusión $^{(6,12,16)}$. Clínicamente se caracteriza por presentar disuria, urgencia miccional, nicturia, 
polaquiuria y dolor pélvico ${ }^{(6,12,15,16)}$. Esta etapa es usualmente autolimitada y raramente persiste por un periodo mayor a los 3 meses postradiación ${ }^{(12)}$.

En fases tardías, los síntomas pueden resurgir desde los 6 meses hasta décadas después de haber recibido las dosis de radiación y los daños causados por la terapia son irreversibles ${ }^{(6,12,15,16)}$. En estos casos, la hematuria es el componente principal del cuadro clínico y puede variar desde microhematuria asintomática hasta macrohematuria que puede poner en riesgo la vida del paciente ${ }^{(2,8,12,14,15)}$. En condiciones avanzadas, se forman coágulos que llegan a obstruir el tracto de salida de la vejiga con lo que eventualmente causan retención urinaria ${ }^{(2,12)}$. Es por esta razón, y por la transformación fibrótica a nivel celular que disminuye la capacidad vesical, que se observan los mismos síntomas urinarios de la fase aguda ${ }^{(6,8,12)}$. Además, la retención de orina eventualmente puede facilitar procesos de sepsis urinaria, perforación de la pared vesical, fistulización e insuficiencia renal ${ }^{(7,10,11,15)}$.

\section{Diagnóstico y Evaluación}

Debido a que las características clínicas de la cistitis postradiación son inespecíficas, el diagnóstico debe realizarse mediante la exclusión de otras causas de hematuria y síntomas urinarios en el contexto de un paciente que previamente haya recibido radioterapia. Las principales entidades a descartar son infección del tracto urinario, procesos neoplásicos y urolitiasis ${ }^{(1,6,9,12,}$ 15).

La evaluación inicial debe incluir el examen general de orina con su respectivo análisis citológico y el urocultivo, para así excluir diagnósticos infecciosos $\mathrm{y}$ de malignidad. A continuación, se deben obtener diversos estudios imagenológicos de todo el tracto urinario con el fin de evaluar la integridad de las estructuras que lo componen y descartar la presencia de otras etiologías que sean responsables de la hematuria. Dentro de los estudios se incluyen la urografía por tomografía, la urografía miccional, el ultrasonido convencional y la citoscopía ${ }^{(1,6,7,9,10,}$ 12, 14). Este último es el que mayor utilidad proporciona a la hora de realizar el diagnóstico definitivo, ya que permite observar la presencia de edema, eritema y atrofia de la mucosa vesical, telangectasias, úlceras sangrantes, fibrosis $\mathrm{y}$ en casos más graves necrosis y fistulización. En caso de identificarse estas alteraciones, se debe correlacionar el área en el que se localizan con aquella que recibió radiación y en caso de que sean las mismas se podrá confirmar la etiología. Durante este procedimiento es posible obtener una biopsia del tejido afectado en caso de que haya dudas sobre el diagnóstico o si existe la sospecha de una presencia o recurrencia tumoral, teniendo en cuenta el potencial riesgo de perforar una órgano que ya ha sido previamente irradiado $^{(6,7,9,12)}$.

\section{Manejo y Tratamiento}

Dentro de las primeras intervenciones a realizar en estos pacientes, se debe incluir una adecuada hidratación intravenosa para garantizar la estabilidad hemodinámica y forzar la diuresis ${ }^{(4,9,}$ 10). Al mismo tiempo es necesario colocar una sonda vesical para la irrigación intermitente o continua de la vejiga con solución salina hasta que la orina se aclare por completo. Esto también permite la eliminación de coágulos en caso de que se hayan formado ${ }^{(4,11-13)}$. Si el sangrado ha sido lo suficiente profuso o prolongado como para provocar anemia e inestabilidad hemodinámica, la transfusión sanguínea deberá ser empleada ${ }^{(6,7,9,10,12)}$.

La mayoría de pacientes que se presentan con esta patología van a responder adecuadamente al manejo conservador y tendrán una resolución satisfactoria de la hematuria. En aquellos casos en los que esto no suceda, se podrán implementar gradualmente diferentes modalidades terapéuticas según la respuesta clínica que se presente ante cada una.

\section{Terapias sistémicas}

\section{Ácido aminocapróico}

Actúa como inhibidor competitivo de la plasmina y el plasminógeno, con lo cual se disminuye la actividad fibrinolítica de la uroquinasa urinaria ${ }^{(4,}$ $10,13,14)$. Su administración puede ser oral ya que es rápidamente absorbida y el $80 \%$ se secreta sin modificaciones en la orina; la dosis en este caso es de $100-150 \mathrm{mg} / \mathrm{kg} / \mathrm{d}$ por un máximo de 21 días $^{(10,14,15)}$. En caso de preferir la vía parenteral, se administra un total de 4-5 g IV durante la primera hora seguido de $1 \mathrm{~g}$ por hora en infusión 
continua. La mayor desventaja del medicamentos es la formación de coágulos que no pueden ser evacuados fácilmente, por lo que no se recomienda una dosis mayor de $12 \mathrm{~g}$ diarios ya que además se ha demostrado el aumento en el riesgo de eventos tromboembólicos ${ }^{(4)}$. Está contraindicado en pacientes con discrasias sanguíneas o con sangrados digestivos activos ${ }^{(10,}$ 15).

\section{Estrógenos conjugados}

Se han utilizado por su efecto en la disminución de la fragilidad de la microvasculatura, por su acción moduladora sobre la respuesta inmune celular y las citoquinas, y por la estimulación de la actividad de las células endoteliales ${ }^{(1,4,6,7,10,12 \text {, }}$ 15). La recomendación consiste en iniciar tratamiento con $6 \mathrm{mg} /$ día repartido en 3 dosis e ir aumentando progresivamente hasta llegar a 12 $\mathrm{mg} /$ día o al control de la hematuria. La respuesta de ausencia de sangrado se ha observado entre las 8 horas y los 7 días, y se recomienda mantener la terapia entre 5-16 semanas con dosis descendientes $^{(10,}{ }^{15)}$. Dentro de los efectos adversos se encuentran la hipercoagulabilidad, la toxicidad hepática y el aumento de riesgo de cáncer hormonodependiente, por lo que es necesario realizar determinación de enzimas hepáticas y asegurar que no haya contraindicaciones de origen ginecológico a pesar de que su presentación sea poco frecuente ${ }^{(6,}$ $8,10,15$.

\section{Pentosan polisulfato}

Un polisacárido semisintético que actúa como precursor de glicosaminoglicanos, por lo que tiene la capacidad de adherirse al epitelio vesical y reemplazar la superficie dañada por la radiación $^{(7,}{ }^{12)}$; esto evita la exposición de la vasculatura y la invasión bacteriana. Al mismo tiempo, tiene propiedades antiinflamatorias gracias a la inhibición de mastocitos y a la disminución de la producción del factor nuclear $\mathrm{KB}^{(4,10,15)}$. La dosis sugerida es de $100 \mathrm{mg} \mathrm{VO}$ cada 8 horas por un período de hasta 3 meses., obteniendo resultados favorables desde las primeras 8 semanas de iniciado el tratamiento. La escasez de efectos secundarios, la ausencia de interacciones con otros tratamientos y la relativa rapidez de los resultados lo colocan como uno de los medicamentos de primera línea en esta patología ${ }^{(6,9,15)}$.

\section{WF10}

Este compuesto es una dilución 1:10 del tetraclorodecaóxido, formulado así para poder ser utilizado por vía intravenosa. Su mecanismo de acción consiste en promover las funciones inmunológicas y de reparación del tejido mediante la activación de macrófagos, contrarrestando de esta manera el proceso inflamatorio producto de la radiación ${ }^{(1,4,6,7,10,12)}$. Algunos efectos adversos incluyen náuseas, cefaleas y anemias transitorias ${ }^{(6)}$.

\section{Factor VIIa recombinante}

Promueve la formación de coágulos de fibrina en sitios de lesión vascular para crear un complejo con el factor tisular expuesto y así actuar sobre las plaquetas activadas, intentando lograr una hemostasia adecuada. Si bien está aprobado para el tratamiento de hemorragias incoercibles de diferentes etiologías (anticoagulación oral, trombocitopenias, traumatismos graves) no lo está para la cistitis hemorrágica, por lo que su uso sería fuera de indicación. La dosis recomendada es de $90 \mathrm{mg} / \mathrm{kg}$, pudiéndose administrar una segunda dosis 20 minutos después si no se logra el resultado esperado ${ }^{(10,15)}$. A pesar de que no se han observado efectos secundarios importantes, sí se ha determinado que la respuesta suele ser temporal ${ }^{(8)}$.

\section{Terapias Intravesicales}

\section{Alumbre}

Mediante su acción astringente causa que las proteínas precipiten en los sitios de sangrado de la superficie del urotelio, permitiendo que este se contraiga, que disminuya la permeabilidad capilar, y que se favorezca la vasoconstricción. Como resultado se logra una disminución del edema local, de la inflamación y de la

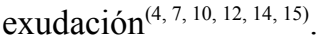

Típicamente, se utiliza una solución de alumbre al $1 \%$ creada al disolver $50 \mathrm{~g}$ de alumbre en $5 \mathrm{~L}$ de agua estéril, y se irriga la vejiga a una 
velocidad de $250-300 \mathrm{~mL} / \mathrm{h}^{(4,10,14,15)}$. Se debe tener cuidado al momento de realizar la irrigación ya que si el sangrado es abundante se pueden crear coágulos grandes que obstruyan el tracto de salida y más bien produzcan distensión y mayor sangrado ${ }^{(4,7,12)}$.

Los efectos secundarios son raros y comúnmente solo consisten de síntomas irritativos locales que pueden ser controlados con medicamentos anticolinérgicos. A nivel sistémico, se ha descrito en pacientes con insuficiencia renal la aparición de encefalopatía, malestar general, trastornos del habla, demencia y convulsiones como efecto de la toxicidad del aluminio acumulado ${ }^{(10,12,14,15)}$.

\section{Ácido hialurónico}

Repone temporalmente la capa deficiente de glicosaminoglicanos del urotelio vesical, estimula la renovación del tejido conjuntivo y logra facilitar el restablecimiento de las células epiteliales $^{(1,8,10,15)}$.

El tratamiento consiste en utilizar una dosis de $40 \mathrm{mg}$ diluidos en $50 \mathrm{~mL}$ de solución fisiológica e instilar por al menos 20-30 minutos. Esto se realiza una vez por semana por un mes y posteriormente una vez al mes hasta que se logren controlar los síntomas ${ }^{(9,10,15)}$. No se ha reportado toxicidad con este fármaco y es muy bien tolerado, logrando una disminución sostenida de los diferentes síntomas y signos de la cistitis incluyendo la hematuria ${ }^{(1,8,9,11)}$.

\section{Formalina}

Provoca la precipitación de proteínas celulares en la mucosa vesical causando la oclusión y fijación de los tejidos telangectásicos y de los capilares, de manera que cesen el sangrado $y$ la exudación ${ }^{(1,4,7,10-12,14)}$. Típicamente se inicia la administración con concentraciones bajas (de 1$2 \%$ ) durante no más de 15 minutos $\mathrm{y}$ asegurándose que la presión intravesical no supere los $15 \mathrm{cmH} 2 \mathrm{O}$.

Posteriormente se debe irrigar de manera continua con solución salina por 12-48 horas debido a los potenciales efectos tóxicos del tratamiento $^{(4,6,10,14,15)}$. Debido a que esta solución es cáustica, la instilación se realiza bajo anestesia espinal o general y es imperativo descartar la presencia de reflujo vesicoureteral o de perforaciones por medio de la citoscopía o cistografía. También se debe proteger el área genital con vaselina para evitar la irritación de la piel y mucosa en caso de que hubiera contacto con la formalina ${ }^{(1,4,6,7,9,10,14,15)}$.

A pesar de estas medidas, la toxicidad asociada con el procedimiento puede causar diversas complicaciones menores y mayores. Es común que se de una fibrosis del epitelio la cual puede llevar a disminución de la contractibilidad vesical y posteriormente a urgencia e incontinencia urinaria. También se han documentado casos de obstrucción ureterovesical que pueden evolucionar a hidronefrosis y llevar a cuadros de fallo renal. Otras complicaciones de menor frecuencia pero mayor seriedad son la necrosis tubular aguda, las fístulas vesicoileales y vesicovaginales, la disyunción vesical, miocardiopatías tóxicas e incluso la muerte ${ }^{(1,4,6-12}$ ${ }^{14,15)}$. Por estas razones, y a pesar de las tasas de éxito tan altas, el uso terapéutico de la formalina se debe restringir a aquellos casos de hemorragia vesical intratable que no hayan respondido a otros tratamientos $^{(4,6,7,10,11)}$. Algunos autores han comprobado la efectividad de colocar, por periodos cortos, compresas impregnadas con formalina sobre los sitios de sangrado, logrando menos complicaciones y resultados favorables similares $^{(6,9,12)}$.

\section{Carboprost}

Este análogo de la prostaglandina F2alfa induce la contracción del músculo liso vascular y la agregación plaquetaria, al mismo tiempo que reduce el edema y la respuesta inflamatoria ${ }^{(4,8,10}$, 12). Se ha observado un aumento en la frecuencia y urgencia urinaria como efectos secundarios del tratamiento $^{(6)}$.

\section{Ácido aminocapróico}

Se realiza una irrigación vesical continua, al disolver $200 \mathrm{mg}$ del medicamento por cada litro de solución salina, y se mantiene hasta 24 horas después de que la hematuria desaparezca ${ }^{(6,14)}$.

\section{Nitrato de plata}

Produce una coagulación química en los sitios de sangrado al utilizar una solución al $0.5-1 \%$ e irrigar por 10-20 minutos $^{(4,6,14)}$. 


\section{Extracto planetario}

Gracias a la presencia de factores angiogénicos y de crecimiento en este tejido, se favorece la epitelización de úlceras vasculares ${ }^{(1,7,10,12)}$. Las prostaglandinas ayudan al disminuir el edema y la respuesta inflamatoria, tal como lo hacen en las mucosas gástricas o yeyunales ${ }^{(7)}$.

\section{Oxigenoterapia Hiperbárica}

La administración de oxígeno en cámaras hiperbáricas aumenta la tensión de oxígeno en el tejido, facilitando la cicatrización de las áreas afectadas $^{(1,7,12,14)}$. En el caso de la cistitis postradiación, se favorece la angiogénesis capilar, se promueve la formación de tejido de granulación y se optimiza la función inmune a nivel celular. Igualmente, se disminuye el edema al inducir la vasoconstricción y se frenan los procesos de infiltración leucocitaria y de $\left.\operatorname{necrosis}^{(1,} 6-8,10,12,13,15\right)$. Los protocolos varían según los centros, pero generalmente se administra $\mathrm{O}_{2}$ al $100 \%$ en cámaras a 2-3 atmósferas de presión durante 90 minutos. Este proceso se realiza una vez al día, 5 veces por semana hasta completar en promedio 30 sesiones, aunque esto va a depender de la respuesta clínica que se observe ${ }^{(1-3,6,9,10,12,15)}$. La terapia suele ser muy bien tolerada y efectiva sin importar los tratamientos previos a los que se haya visto expuesto el paciente, y con la ventaja de que no tiene efectos adversos sobre la estructura o funcionalidad vesical. Los resultados son dependientes de factores como la edad, la dosis de radiación recibida y la severidad de la hematuria al momento de inicio de las sesiones ${ }^{(1,}$ $2,4,10,12,15)$. Existen pocas complicaciones que pueden surgir con la oxigenoterapia hiperbárica, siendo las más comunes los barotraumas auditivos. Otras más raras son el neumotórax y la toxicidad del sistema nervioso central o de los pulmones $^{(6,10,12,14)}$.

\section{Cistoscopía y coagulación}

Ante la falla del manejo conservador, se puede recurrir al uso de diferentes técnicas mínimamente invasivas que permitan la coagulación intravesical por medio de la cistoscopía. La diatermia, el gas argón y el láser son los métodos de mayor empleo. El primero utiliza una corriente eléctrica para producir calor y coagular el tejido hemorrágico directamente ${ }^{(7,}$

12); el segundo utiliza el gas como medio de conducción de la electricidad y así se evita el contacto con la lesión, disminuyendo el riesgo de ruptura de la pared vesical ${ }^{(4,6,10)}$; por último, el láser (KTP) tiene la capacidad de ser absorbido selectivamente por la hemoglobina logrando una hemostasia más eficaz sin afectar al tejido circundante de manera importante ${ }^{(4,6)}$.

\section{Embolización arterial}

Otra de las opciones intervencionistas que existe para corregir la hematuria refractaria es la embolización selectiva de las arterias que alimentan al tejido vesical. En primera instancia se describió la embolización unilateral de la rama anterior de la arteria ilíaca interna, sin embargo la importancia de la circulación colateral obliga a realizar un abordaje bilateral ${ }^{(4,6,10,14)}$. Es común que aparezca como consecuencia el dolor glúteo por isquemia en aquellos casos en los que además se haya ocluido la arteria glútea superior $^{(4,6,10,14,15)}$. Actualmente se prefiere ejecutar un procedimiento súper selectivo en el que se buscan vasos más pequeños y específicos para así lograr mejores resultados y menos complicaciones $^{(4,6,7,15)}$.

\section{Cirugía}

La intervención quirúrgica debe ser el último recurso en el tratamiento de la cistitis hemorrágica, una vez que todos los otros métodos hayan fallado en el control de la hematuria. Cualquiera que sea la opción empleada implica un grado de dificultad importante, al tratarse principalmente de pacientes mayores con varias comorbilidades y al tener que actuar en sitios anatómicos que han sido dañados por la radiación $\left.{ }^{(4-6,8}, 10,12,14\right)$. La escogencia del tipo de derivación urinaria será determinada por las características de cada paciente, incluyendo su grado de autonomía, la patología de base y el pronóstico de sobrevida ${ }^{(15)}$.

Dentro de los procedimientos recomendados se encuentran la nefrostomía percutánea bilateral (con o sin oclusión ureteral), la cistostomía suprapúbica, y el conducto intestinal ${ }^{(1,4,6,8,1214)}$. El objetivo principal de estos tipos de derivación es el de evitar la exposición de áreas hemorrágicas a la uroquinasa para que se pueda 
dar una adecuada hemostasia del tejido vesical ${ }^{(4,}$ 14).

Dadas las complicaciones asociadas con tales métodos, y a que se han documentado tasas de morbimortalidad perioperatorias muy similares, alguna literatura recomienda realizar concomitantemente una cistectomía radical ya que es esta la única solución definitiva para controlar la hemorragia refractaria ${ }^{(1,6,8,11,12,14)}$.

\section{CONCLUSIONES}

La cistitis hemorrágica postradiación es una entidad poco frecuente pero cuya incidencia irá en aumento conforme se envejezca la población y se utilice más la radioterapia en el tratamiento de las diferentes patologías oncológicas pélvicas. A pesar de las múltiples opciones terapéuticas disponibles, es necesario realizar más y mejores estudios que comparen estrategias e identifiquen cuál es el mejor manejo. Solamente de esta manera se logrará confeccionar guías y protocolos universales para disminuir la morbimortalidad de manera efectiva y eficiente.

\section{BIBLIOGRAFÍA}

1. Browne C Davis NF Mac Craith E et al. A Narrative Review on the Pathophysiology and Management for Radiation Cystitis. Advances in Urology. 2015;2015:1-7.

2. Oliai C Fisher B Jani A et al. Hyperbaric Oxygen Therapy for Radiation-Induced Cystitis and Proctitis. Int J Radiat Oncol Biol Phys. 2012;84(3):733-740.

3. Gallego Vilar D García Fadrique G Povo Martín IJ. Oxigenoterapia hiperbárica $(\mathrm{OHB})$ en el manejo de la cistitis hemorrágica radioinducida. Arch. Esp. Urol. 2011;64(9):869-874

4. Alesawi AM El-Hakim A Zorn KC Saad F. Radiation-induced hemorrhagic cys- titis. Curr Opin Support Palliat Care. 2014 Sep;8(3):235-240.

5. Linder BJ Tarrell RF Boorjian SA. Cystectomy for Refractory Hemorrhagic Cystitis: Contemporary Etiology, Presentation and Outcomes. $\mathrm{J}$ Urol. 2014;192(6):1687-1692.

6. Liem X Saad F Delouya G. A Practical Approach to the Management of Radiation-Induced Hemorrhagic Cystitis. Drugs. 2015;75(13):1471-1482.

7. Denton AS Clarke N Maher J. Non-surgical interventions for late radiation cystitis in patients who have received radical radiotherapy to the pelvis. Cochrane Database Syst Rev. 2002; (3):CD001773.

8. Payne H Adamson A Bahl A et al. Chemicaland radiation-induced haemorrhagic cystitis: current treatments and challenges. BJU Int. 2013;112(7):885897

9. Mendenhall WM Henderson RH Costa JA et al. Hemorrhagic Radiation Cystitis. Am J Clin Oncol. 2015;38(3):331336.

10. López González MH Cruz Arévalo DA Valero Pulido JC. Manejo de la hematuria intratable en cistitis posterior a radioterapia. Reporte de dos casos y revisión de la literatura. Rev Urol Colomb. 2013;XXII(3):53-60.

11. Zwaans BMM Chancellor MB Lamb LE. Modeling and Treatment of Radiation Cystitis. Urology. 2016;88:14-21.

12. Smit SG Heyns CF. Management of radiation cystitis. Nat Rev Urol. 2010;7(4):206-214.

13. Haldar S Dru C Bhowmick NA. Mechanisms of hemorrhagic cystitis. Am J Clin Exp Urol. 2014;2(3):199-208. 
14. Linder BJ Boorjian SA. Management of emergency bleeding, recalcitrant clots and hemorrhagic cystitis. AUA Update Series. 2015;34(lesson 3).

15. Martínez-Rodríguez R Areal Calama J Buisan Rueda et al. Guía práctica para

\section{CONFLICTO DE INTERÉS Y/O AGRADECIMIENTOS}

Los autores declaran que no existió ningún conflicto de interés en el presente artículo. el manejo y tratamiento ante la cistitis rádica. Actas Urol Esp. 2010;34(7):603-609.

16. Zhou M Netto G Epstein JI. Uropathology. Saunders USA. 1ed, 2012 